\title{
Sommeil, éveil et cognition au cours du temps
}

\author{
Gilles VANDEWALLE
}

\author{
Université de Liège \\ GIGA-Research - Cyclotron Research Centre/In Vivo Imaging Unit \\ Liège, Belgique
}

L'être humain est un animal diurne qui a typiquement un seul épisode de veille et un seul épisode de sommeil par cycle de $24 \mathrm{~h}$. Ce simple fait est tout à fait remarquable quand on sait les nombreux changements dynamiques qui ont court dans le cerveau pendant l'éveil et le sommeil. Sur une échelle temporelle de $24 \mathrm{~h}$, être éveillé est en effet associé à des modifications moléculaires, cellulaires et systémiques du cerveau humain qui ont un impact négatif sur la cognition. Or, nous sommes capables de rester éveillés pendant une journée entière $(\sim 16 \mathrm{~h})$ en maintenant des performances cognitives relativement stables. Ce n'est que si nous prolongeons l'éveil au-delà d'une journée normale, lorsque nous restons éveillés alors qu'habituellement nous dormons (typiquement la nuit), que nos capacités cognitives sont compromises et nos performances diminuent significativement. Cet aspect «non-linéaire » de notre fonctionnement cognitif, le fait que la diminution des capacités cognitives ne soit pas plus progressive, reflète l'implication du système circadien, au côté de l'homéostasie du sommeil, dans la régulation du fonctionnement cérébral.

L'homéostasie du sommeil est un « sablier » qui garde une trace du temps éveillé et qui accumule progressivement la pression de sommeil (le besoin de dormir). Cette accumulation est dissipée durant le sommeil. Le système circadien, quant à lui, organise les différents processus physiologiques dans le temps et sépare les processus incompatibles. Au niveau du fonctionnement cognitif cérébral ces deux processus interagissent de telle façon que le système circadien envoie un signal d'éveil la journée qui contre l'accumulation progressive de pression de sommeil au cours de l'éveil. Ce signal est maximal en soirée durant la «zone de maintien de l'éveil » située 1 à $2 \mathrm{~h}$ avant l'heure habituelle de coucher. C'est ce signal d'éveil qui permet une cognition relativement stable durant $\sim 16 \mathrm{~h}$ consécutives. La nuit, le signal circadien favorise le sommeil et va à l'encontre de la diminution progressive du besoin de dormir durant le sommeil pour permettre un épisode consolidé de sommeil, même en fin de nuit. Ce même signal de sommeil va amplifier les effets délétères de la pression de sommeil et avoir un impact négatif sur la cognition, si un individu reste éveillé la nuit quand, normalement, il dort. 
Il existe une variabilité inter-individuelle importante dans la rapidité avec laquelle la pression de sommeil s'accumule ainsi que dans la période d'un cycle circadien individuel (en moyenne $24,2 \mathrm{~h}$, soit un peu plus que $24 \mathrm{~h}$, mais pouvant aller de $\sim 23.8 \mathrm{~h}$ à $\sim 24.6 \mathrm{~h}$ ). Et il semble que cette variabilité soit responsable, en partie au moins, des différences susceptibilités au manque de sommeil présentes dans la population (certains individus résistent mieux que d'autres au manque de sommeil et/ou semblent avoir besoin de moins de sommeil que d'autres) ainsi que des différents « chronotypes » qui caractérisent les individus (être « du matin » ou « du soir »).

De nombreuses études ont utilisé l'électroencéphalogramme (EEG) ainsi que diverses tâches ou tests cognitifs pour établir ce scénario. Les recherches en neuroimagerie mises au point spécialement pour comprendre les mécanismes cérébraux qui sous-tendent l'interaction entre l'homéostasie du sommeil et le système circadien sont plus rares et sont une des spécificités de l'Université de Liège (ULg). Ces recherches montrent tout d'abord que la capacité de recruter des régions cérébrales sous-corticales, qui sont essentielles à la régulation de l'éveil et du sommeil, est un élément clé à la bonne réalisation de processus cognitifs après plusieurs heures d'éveil, et après une nuit sans sommeil. D'autres recherches démontrent ensuite que l'influence de l'homéostat du sommeil et du système circadien affecte le fonctionnement basique des neurones (leur réactivité), présente des spécificités locales (l'impact varie d'une région cérébrale à l'autre) et est détectée pratiquement dans toutes les régions du cerveau. Le décours temporel de notre activité cérébrale cognitive au cours des $24 \mathrm{~h}$ d'une journée est donc dicté par l'interaction entre homéostat et système circadien au cours de l'éveil et du sommeil.

Au-delà de cette échelle temporelle, une autre échelle de temps pourrait affecter notre cognition : les 365 jours et les 4 saisons d'une année. Des rythmes saisonniers ou circannuels ont été identifiés dans tout le règne animal et l'être humain n'est pas une exception. Il est d'ailleurs intéressant de noter que cette saisonnalité est détectée surtout au niveau de fonctions dépendant du cerveau. Au-delà d'une influence sur le système immunologique et sur le taux de natalité et de décès, les variations saisonnières avérées chez l'être humain concernent surtout l'humeur et les troubles de l'humeur. Ces variations d'humeur sont liées à des troubles du sommeil et de l'éveil ainsi qu'à des troubles cognitifs. La saisonnalité des fonctions cognitives cérébrales humaines reste néanmoins peu explorée chez des individus en bonne santé. Une recherche, menée à l'ULg, dans des conditions contrôlant de nombreux biais potentiels, a cependant démontré que l'activité cérébrale cognitive mesurée par imagerie par résonance magnétique (IRM) fluctuait significativement avec les saisons. Cette fluctuation semble dépendre par ailleurs du processus cognitif mis en jeu: des processus attentionnels plus basiques semblaient plus liés à la photopériode, alors que des processus cognitifs plus complexes serait plus liés à d'autres facteurs non établis, ce qui pourrait engendrer un décalage de phase de plusieurs mois dans la rythmicité annuelle de certaines fonctions cognitives. 
L'implication de ces découvertes n'est pas encore claire, mais elles pourraient constituer le terrain sur lequel les troubles de l'humeur viennent se greffer chez des personnes plus susceptibles aux variations saisonnières que d'autres. Il est enfin intéressant de noter que la recherche chez l'animal a permis de montrer que la machinerie moléculaire de «l'horloge saisonnière » ou «circannuelle » est en partie commune au système circadien. Le lien entre sommeil, éveil et cognition dépend donc aussi d'une échelle de temps annuelle, bien plus longue que l'échelle de $24 \mathrm{~h}$ à laquelle on l'étudie.

A nouveau, au-delà d'une année, un autre temps est à prendre en considération ici: celui une vie. La petite enfance et l'adolescence sont sans nul doute des périodes de grands changements cognitifs ainsi qu'au niveau des besoins de sommeil et de la rythmicité circadienne. Des modifications importantes ont également lieu au cours de l'âge adulte. Ainsi, dès l'âge moyen, soit entre 40 et 60 ans, le sommeil devient significativement plus fragmenté, moins profond et les individus ont tendance à se coucher et à se lever plus tôt. Ces modifications sont vraisemblablement dues à une croissance moins rapide de la pression de sommeil au cours des périodes d'éveil et à une «avance de phase » de l'horloge circadienne. Une des résultantes un peu inattendue de ces changements est, qu'à court terme en tout cas, les personnes plus âgées souffrent relativement moins du manque de sommeil, et résistent mieux par exemple à une nuit sans sommeil. Des recherches menées à Bâle et à Liège montrent tout d'abord que cet impact délétère moins important d'une période d'éveil nocturne, pendant la période habituelle de sommeil, se reflète par une variation moins importante de la réactivité des neurones au cours de l'éveil et particulièrement pendant la nuit. Ces recherches indiquent par ailleurs que la capacité des personnes plus âgées à recruter des régions sous-corticales importantes pour l'éveil et le sommeil, soit un facteur important de la diminution relative de l'effet négatif du manque de sommeil chez les individus plus âgés.

Dans une société qui sacrifie la durée et la qualité du sommeil pour étendre de plus en plus les activités d'éveil, qu'elles soient professionnelles (travail de nuit, décalage horaire, horaires de travail prolongés, etc.), sociales ou récréationnelles (TV, etc.), dans une société qui exige de l'efficacité toute l'année, et dans une société vieillissante, il est important de comprendre les mécanismes cérébraux qui sous-tendent le lien entre cognition, sommeil et éveil et sa variabilité afin de pouvoir mettre au point des stratégies efficaces de prévention et de gestion des problèmes de sommeil et de vigilance qui sont monnaie courante actuellement. Les recherches présentées ici contribuent à cette compréhension.

L'activité cognitive dépend donc de la qualité et de la durée du sommeil et de l'éveil qui la précèdent mais aussi du moment de l'année et de la vie auxquel elle est effectuée. Des échelles temporelles bien plus courtes n'ont pas été abordées dans ce texte, tels que, par exemple, la génération de potentiels d'action, qui se régule en quelques dizaines de millisecondes, ou encore les questions de plasticité neuronale et d'apprentissage qui font intervenir des processus pouvant 
se dérouler en quelques minutes, quelques heures, quelques jours, voire plusieurs années. Ces échelles de temps ne constituaient pas le thème de cet exposé, mais méritent tout autant d'attention que les choses abordées ici.

\section{Références}

1. Dijk, D. J. \& Archer, S. N. PERIOD3, circadian phenotypes, and sleep homeostasis. Sleep Med. Rev. 14, 151-160 (2010).

2. Gaggioni, G., Maquet, P., Schmidt, C., Dijk, D. \& Vandewalle, G. Neuroimaging, cognition, light and circadian rhythms. Front. Syst. Neurosci. 8, 126 (2014).

3. Ly, J. Q. M., Gaggioni, G., Chellappa, S. L., Papachilleos, S., Brzozowski, A., Borsu, C., Rosanova, M., Sarasso, S., Middleton, B., Luxen, A., Archer, S. N., Phillips, C., Dijk, D.J., Maquet, P., Massimini, M. \& Vandewalle, G. Circadian regulation of human cortical excitability. Nat Commun In Press, 11828 (2016).

4. Wirz-Justice, A., Benedetti, F., Berger, M., Lam, R. W., Martiny, K., Terman, M. \& Wu, J. C. Chronotherapeutics (light and wake therapy) in affective disorders. Psychol. Med. 35, 939-944 (2005).

5. Muto V, Jaspar M, Meyer C, Kussé C, Chellappa SL, Degueldre C, Balteau E, Shaffii-Le Bourdiec A, Luxen A, Middleton B, Archer SN, Phillips C, Collette F, Vandewalle G, Dijk DJ, M. P. Local Modulation of Human Brain Responses by Circadian Rhythmicity and Sleep Debt. Science (80-. ). in press, (2016).

6. Meyer, C., Muto, V., Jaspar, M., Kussé, C., Lambot, E., Chellappa, S. L., Degueldre, C., Balteau, E., Luxen, A., Middleton, B., Archer, S. N., Collette, F., Dijk, D.-J., Phillips, C., Maquet, P. \& Vandewalle, G. Seasonality in human cognitive brain responses. Proc. Natl. Acad. Sci. U. S. A. 113, 3066-3071 (2016).

7. Carrier, J., Viens, I., Poirier, G., Robillard, R., Lafortune, M., Vandewalle, G., Martin, N., Barakat, M., Paquet, J. \& Filipini, D. Sleep slow wave changes during the middle years of life. Eur. J. Neurosci. 33, 758-766 (2011).

8. Cedernaes, J., Osorio, R. S., Varga, A. W., Kam, K., Schiöth, H. B. \& Benedict, C. Candidate Mechanisms Underlying the Association Between Sleep-Wake Disruptions and Alzheimer's Disease. Sleep Med. Rev. 1-10 (2016). doi:10.1016/j.smrv.2016.02.002 